\title{
Registros de enfermagem: reflexões sobre o cotidiano do cuidar
}

\author{
Nursing records: reflections on daily care \\ Mayara Mota Araujo, Samanta Oliveira da Silva Diniz²,3, Paulo Sérgio da Silva 2,4 \\ ${ }^{1}$ Maternidade Escola, Universidade Federal do Rio de Janeiro (UFRJ) - Rio de Janeiro (RJ), Brasil. \\ ${ }^{2}$ Programa de Pós-Graduação em Enfermagem e Biociências, Universidade Federal do Estado do Rio de Janeiro (UNIRIO) - Rio de Janeiro (RJ), Brasil. \\ ${ }^{3}$ Escola de Ciências da Saúde, Universidade do Grande Rio (UNIGRANRIO) - Duque de Caxias (RJ), Brasil. \\ ${ }^{4}$ Curso de Graduação em Enfermagem, Centro Universitário Serra dos Órgãos (UNIFESO) - Teresópolis (RJ), Brasil.
}

DOI: http://dx.doi.org/10.7322/abcshs.v42i3.920

\section{RESUMO}

O presente ensaio teve por objetivo refletir sobre as considerações conceituais dos registros de enfermagem em sua dimensão assistencial. Trata-se de uma revisão narrativa que discutiu os registros de enfermagem sob o ponto de vista teórico e contextual. A síntese crítica foi produzida a partir das dissertações e teses vinculadas à Coordenação de Aperfeiçoamento de Pessoal de Nível Superior (CAPES) com aderência à área de Enfermagem Fundamental; artigos atuais com mais de cem citações pesquisados no Google Acadêmico, sobretudo os que discorrem sobre o núcleo significativo para a construção das reflexões; e registros de enfermagem. O material produzido foi submetido à análise de conteúdo de Bardin e agrupado em uma unidade descritiva intitulada "mensagens científicas dos registros de enfermagem para o cotidiano do cuidar". Nessa pesquisa estão contidas as análises reflexivas sobre as formas e os conteúdos dos registros provenientes das situações de cuidar ocorridas a partir do encontro estabelecido entre a enfermagem e o paciente nos diversos cenários do cuidado. As (in)conclusões dos materiais analisados apontaram para registros de enfermagem cifrados, ilegíveis, presença de rasuras, erros ortográficos, espaço em branco entre as anotações, conteúdos que privilegiam o saber biomédico e uso indiscriminado de abreviaturas e siglas.

Palavras-chave: enfermagem; cuidados de enfermagem; registros de enfermagem.

\begin{abstract}
The purpose of the present essay was to reflect on the conceptual considerations of nursing records in care dimension. This is a narrative review on nursing records from a theoretical and contextual point of view. The critical synthesis was produced from dissertations theses linked to the Coordination of Improvement of Higher Education Personnel (CAPES) in the field of Fundamental Nursing; current articles with more than 100 citations in Google Scholar, especially those addressing a significant core for reflection; and nursing records. The material produced was submitted to Bardin's content analysis and grouped in a descriptive unit entitled "scientific messages from daily care nursing records". It contains reflective remarks on the forms and contents of records from care situations resulting from the nurse-client meetings in different settings. The (in)conclusions of the materials point out to enciphered, unreadable nursing records full of erasures, spelling mistakes, blank spaces between annotations, and contents that privilege biomedical knowledge and the indiscriminant use of abbreviations and acronyms.
\end{abstract}

Keywords: nursing; nursing care; nursing records. 


\section{INTRODUÇÃO}

As reflexões postas estão alicerçadas nas diretrizes teóricas deixadas pela fundadora da enfermagem moderna, Florence Nightingale. Ela orienta todos os profissionais, sobretudo os enfermeiros, quanto aos aspectos ambientais quando diz que a enfermagem, no desenvolvimento de suas práticas de cuidar, deve significar o uso de ar puro, silêncio, aquecimento, iluminação, limpeza e seleção adequada tanto da dieta quanto da forma de servi-la ${ }^{1}$.

A apropriação dos conceitos relacionados ao ambiente é inerente ao pensamento de que a Enfermagem Fundamental é responsável pela ética profissional, pela observação, pela criatividade e, principalmente, pela qualidade dos registros advindos das práticas de cuidar.

Os debates em torno dos registros escritos pela enfermagem apontam para sua apresentação como um dos principais instrumentos de comunicação para a troca de informações entre as equipes envolvidas no cuidado, cujo objetivo reside em apresentar de forma clara as necessidades do paciente, as condutas clínicas de cuidar que foram implementadas e a avaliação contínua do cuidado prestado. Nesse aspecto, registrar é uma responsabilidade ética e social ${ }^{2}$.

Registros de enfermagem completos delimitam as ações de cuidar desenvolvidas no interior de um processo de trabalho e indicam o modo como os enfermeiros e suas equipes prestam, representam e significam o cuidado de enfermagem que contém indícios reveladores da história e da cultura profissional.

$O$ fato é que os registros de enfermagem são diretamente incididos por um forte apelo para a comunicação escrita de doenças e diagnósticos médicos, enquanto as ditas condutas clínicas de cuidar no domínio da equipe são apresentadas de forma reduzida, cifrada e incompleta ${ }^{3}$.

Nesse contexto, o fazer da enfermagem, sua linguagem e seu saber são ocultados por registros sem conteúdo e sem profundidade, o que dificulta a incorporação de linguagem própria e termos universais entre a equipe que trabalha nessa área.

Inúmeros fatores podem influenciar na qualidade dos registros, dentre os quais se destacam: excesso de pacientes sob a responsabilidade da equipe de enfermagem nos cenários do cuidado; enfermeiros sobrecarregados por atividades de ordem gerencial; inclusão de formulários eletrônicos sem a devida capacitação profissional da equipe; desconhecimento das implicações éticas e legais dos registros; e falta de infraestrutura adequada e equipes bem treinadas e lideradas.

Esses problemas instigam a pensar que é imprescindível refletir sobre os fundamentos da profissão, especificamente aqueles que norteiam os registros dos cuidados desenvolvidos pela enfermagem. A partir dessa relação é traçado o objetivo deste ensaio crítico: refletir sobre as considerações conceituais dos registros de enfermagem em sua dimensão assistencial.

Como não há um enredo listado a priori para essa tarefa, optou-se pela criação de unidades descritivas que discorrem criticamente sobre os registros de enfermagem contextualizados com a prática assistencial desenvolvida nos cenários do cuidado pelo qual os enfermeiros transitam.
Dessa forma, na condição de "ensaístas" e curiosos para compreender os fenômenos por associações ou analogias, rendemo-nos aqui ao formalismo da técnica científica tradicional para experimentar as diversas possibilidades de um vir-a-ser do objeto analisado, nesse caso os registros de enfermagem ${ }^{4}$.

Para tanto, foi assumida a revisão narrativa como caminho a ser percorrido. Esse tipo de contribuição científica tem um papel fundamental para a educação continuada, pois permite ao leitor adquirir e atualizar o conhecimento sobre uma temática específica em curto espaço de tempo. Cabe destacar que essa modalidade de estudo não possui metodologias que permitam a reprodução dos dados nem fornecem respostas quantitativas para questões peculiares, sendo, portanto, considerados qualitativos em sua natureza ${ }^{5}$.

Com base nessas dimensões conceituais, optou-se por definir os materiais a serem consultados nesta revisão da seguinte forma: produções vinculadas ao banco de dissertações e teses da Coordenação de Aperfeiçoamento de Pessoal de Nível Superior (CAPES) com aderência à área de Enfermagem Fundamental; trabalhos consultados com mais de cem citações identificados no Google Acadêmico, sobretudo os que discorrem fundamentalmente sobre os núcleos conceituais deste ensaio; e registros de enfermagem.

A partir desses critérios foi criada a unidade descritiva "mensagens científicas dos registros de enfermagem para o cotidiano do cuidar", destinada à compreensão e discussão do tema na área da Enfermagem.

Nela constam argumentações lógicas sobre as reverberações dos registros de enfermagem no plano assistencial, do qual é apresentada a síntese conclusiva de todo material produzido que seguiu o referencial teórico-analítico de Bardin. Essa técnica de análise consiste em apurar descrições de conteúdo de forma aproximativa e subjetiva para pôr em evidência, com objetividade, a natureza do fenômeno investigado ${ }^{6}$.

\section{Unidade descritiva: mensagens científicas dos registros de enfermagem para o cotidiano do cuidar}

Apresentar de forma descritiva, sintética e lógica os produtos intelectuais que retratam as experiências da enfermagem na ação de registrar foi um desafio. Isso porque a todo instante o pensamento investiga a si mesmo e examina como enfermeiro o caráter de sua própria atividade, estabelecendo os princípios que fundamentam a expressividade do que é habitualmente registrado ${ }^{7}$.

É um pouco disto o que se pretende dizer: a unidade de um registro centraliza em um mesmo campo todos os vestígios que representam os movimentos e gestos cotidianos nas práticas do enfermeiro no cenário de cuidado.

Certamente, os registros manuscritos ou digitados possibilitam a manifestação de inúmeras expressões da enfermagem, que se projetam concretamente sobre as bases teorizantes do cuidado.

Formas e conteúdos de registros de enfermagem em cenário hospitalar denotam a redução de escritas por plantão, desvios no registro de data e horário, ilegibilidade na escrita, abreviaturas, siglas, erros de ortografia, rasuras e espaços em branco entre as anotações ${ }^{2}$. 
Uma regra importante na execução dos registros de enfermagem é a obrigatoriedade da assinatura e da identificação do profissional com o número de registro no Conselho Regional de Enfermagem (COREN) ${ }^{8}$. Um estudo que buscou avaliar os registros da equipe de enfermagem de um hospital escola revelou falhas na identificação do profissional executor das anotações. Os profissionais identificavam-se inadequadamente, apresentando apenas rubrica, sem o número do COREN e sem identificação e informações relacionadas às medidas terapêuticas. Quanto à concisão e à organização, os registros mostraram-se inadequados ${ }^{9}$.

O uso indiscriminado de siglas e abreviaturas não padronizadas aponta para uma forma muito peculiar de anotar. O registro se assemelha a um conjunto de códigos que só podem ser decifrados por profissionais do mesmo cenário de cuidar. Além disso, identifica-se ausência de informações sobre os pacientes cuidados, incoerência e falta de estruturação e de continuidade nos registros realizados pela equipe, comprometendo a sequência das atividades da assistência de enfermagem ${ }^{10}$.

Por outro lado, percebe-se que a introdução de um sistema de linguagem eletrônica modifica os esquemas mentais que os enfermeiros utilizam para dar significado aos dados que observam nos pacientes, conduzindo-os a conclusões diferentes das que chegariam sem o uso padronizado desse sistema $\mathrm{A}$ implementação de uma linguagem unificada possibilita a ampliação dos enfoques dados pelos(as) enfermeiros(as) no cuidado ao paciente, aumentando a ênfase em fenômenos até então pouco documentados ${ }^{11}$.

O que se tem dito sobre a assistência do enfermeiro é que ela é completada quando efetivamente registrada. A profissão exige que ele registre e documente as práticas de cuidar com responsabilidade ética e moral ${ }^{12}$. Nos registros, a ética é enraizada como norteadora dos comportamentos dos enfermeiros e orienta suas ações e atitudes nas cenas do cuidado.

Nesse aspecto, um dos componentes evidenciados sobre os registros diz respeito à enfermagem cuidar de um corpo incompleto. São escassas as anotações relacionadas aos aspectos psicossocioespirituais, o que comprova a despreocupação da enfermagem em registrar o ser na sua totalidade, ou seja, os elementos que vão além do cuidado ao corpo biológico ${ }^{13}$.

Entre os profissionais envolvidos no cuidado, o enfermeiro é o que passa mais tempo com o paciente. Logo, as demandas e necessidades relacionadas a questões que ultrapassam os aspectos físicos, biológicos e patológicos são, muitas vezes, supridas por esses profissionais. Certamente, em sua prática assistencial cotidiana, os enfermeiros convivem com ansiedade, medo, angústia e tensão dos pacientes, trazendo conforto e alívio mediante o toque, a conversa e a escuta. Todavia, não existe documentação desses momentos, talvez por não acreditar-se que sejam merecedores de registro.

Todas as práticas de cuidar merecem e devem ser registradas. Cuidar e registrar são verbos de ação para onde convergem as imagens passadas de um cuidado que têm implicações futuras para o paciente. O que se pretende afirmar é: o corpo cuidado precisa de um registro que contenha elementos suficientes para a efetivação de práticas em saúde que atendam suas necessidades básicas.

É preciso considerar que a escrita pode ser vivida como experiência. Nesse aspecto, é no cenário da prática profissional que o enfermeiro assume a construção de uma narração coletiva ou individual, ou seja, é nesse momento que há uma escrita que registra ações.

Sim, os registros são criados a partir da intensa relação do enfermeiro com o indivíduo que está sendo cuidado e contêm informações adquiridas de acordo com a articulação do ver, ouvir e sentir ${ }^{14}$.

É preciso considerar que esses requisitos vão além dos sinais e sintomas e são aprofundados na subjetividade, ou seja, no que o enfermeiro consegue perceber, ao relatar também os movimentos expressivos, os desejos e as aspirações captadas do corpo do paciente.

Nesse prisma, é reconhecida a necessidade de ampliação no plano da escrita, que deve estar junto a questões específicas da vida dos pacientes que são cuidados, capaz de valorizar a individualidade, os sonhos, as aspirações e as motivações sentidas nos cenários do cuidado.

Nessa situação, quando os enfermeiros não mantêm um registro escrito minuciosamente de todos os cuidados dirigidos ao indivíduo, rompe-se a ligação do processo dinâmico e o agrupamento das relações entre as equipes, logo as ações em saúde ficam comprometidas devido a uma lacuna na comunicação.

Diga-se que as situações de cuidar que acontecem a partir do encontro entre o enfermeiro e o paciente apresentam condições requeridas para alcançar destaque nos registros, os quais alicerçam um conjunto de saberes práticos da profissão.

O campo da escrita recebe contribuições expressivas tanto na produção teórica quanto na prática. $\mathrm{E}$ as mudanças advindas do conhecimento em enfermagem contemporânea nos situam em outro patamar de reflexões e discussões críticas.

É dessa forma que as combinações morais depositam o dever do enfermeiro em um lugar central do discurso ético, permitindo que sua maior grandeza se concentre na imposição, a si mesmo, das obrigações estabelecidas pelo Código de Ética Profissional.

Nesse prisma, o registro é uma das manifestações do cuidado de enfermagem que retratam, a partir da linguagem escrita, uma prática contínua destinada às reforçadas necessidades do indivíduo. Os enfermeiros avaliam esse instrumento como uma das estratégias mais eficientes para continuar a assistência e um espaço correto para a documentação do cuidado. Mas o problema reside no fato de as informações nele contidas serem incompletas, não retratarem as reais condições do paciente e não contribuírem como um indicador de qualidade, o que demonstra sua ineficiência como meio de comunicação ${ }^{15-17}$.

A finalidade dessa forma de comunicação, tanto manuscrita quanto digitada, é divulgar a mensagem com as informações referentes à evolução clínica do paciente, destinada a quem deseja decodificá-la.

Com a ampliação das discussões sobre os achados atuais na temática, constata-se, além das siglas, o uso de abreviaturas não convencionais, nomes comerciais de artefatos e números reduzidos de enunciados de diagnósticos de enfermagem nos registros 
de evolução, mesmo na ausência de um sistema de classificação padronizado institucionalmente ${ }^{18}$.

O que se atesta é a enorme complexidade dos registros em sua relação com a prática profissional de enfermagem. O cuidado, entendido como sistematizado e contínuo, pode ter o seu padrão comprometido quando elementos indispensáveis do histórico, do diagnóstico, do planejamento, da implementação e da avaliação não são detalhadamente registrados.

Considerando que a realização dos registros completos, claros e precisos contribui para a melhoria na qualidade da assistência, favorecendo a continuidade do cuidado e a legitimação do trabalho de enfermagem e ainda que todas as fases da Sistematização da Assistência de Enfermagem (SAE) precisem ser realizadas, evidencia-se a necessidade de acompanhamento mais amiúde dos registros efetuados no dia a dia. Além disso, destaca-se a importância da realização de cursos de educação em serviço para todos os membros da equipe de enfermagem de modo a sensibilizá-los quanto ao valor das informações relacionadas à condição dos pacientes cuidados e, em especial, às ações realizadas para seu atendimento ${ }^{19}$.

$O$ fato é que os registros de enfermagem têm se tornado um dos temas mais relevantes para a categoria profissional na atualidade, pois existe relação direta entre a qualidade das anotações e o cuidado prestado ao paciente de forma segura. Com essas preocupações, é possível destacar esforços para aprimorar e sensibilizar os profissionais de enfermagem quanto à qualidade dos registros, sobretudo em unidades hospitalares.

As orientações científicas sugerem que os registros de enfermagem objetivados na qualidade da assistência e segurança do cuidado devem ser alvo permanente de análises orientadas por protocolos, manutenção de auditorias internas e, especialmente, de atividades educativas mobilizadas pela avaliação criteriosa da Joint Commission International (JCI) ${ }^{20}$.

Somado a isso, cabe a reflexão de desenhos de ferramentas que têm por objetivo potencializar a valorização dos registros clínicos de enfermagem com linguagens próprias e universais para expressar os fenômenos e ações de cuidar desenvolvidas por enfermeiros ${ }^{21}$.

Em suma, atingir a autonomia nos cenários de cuidar passa pela ação de registrar, e esse movimento deve ser realizado com qualidade. Na busca pela melhoria dos registros de enfermagem, estratégias já estão sendo pensadas e operacionalizadas, tais como: atividades educativas em serviço; observação dos registros corpo a corpo no cenário do cuidado; implantação de protocolos; auditorias de prontuários; e desenvolvimento de ferramentas para comunicação nos serviços de saúde.

Mediante o exposto, é fundamental continuar a discussão sobre os registros de enfermagem, sobretudo no cotidiano de cuidar, gerenciar, ensinar e pesquisar, como forma de aprimorar a qualidade das mensagens produzidas sobre os pacientes cuidados nos diversos cenários profissionais em que a saúde e seus desvios são objetos de trabalho dos enfermeiros.

\section{(IN)CONCLUSÃO}

Com a certeza do inacabado, as teses, dissertações e artigos atuais sobre o tema de cunho assistencial dispararam conceitos sobre os registros de enfermagem que retratam a comunicação escrita como alvo de atenção no mundo globalizado, sobretudo quando analisadas as dificuldades da equipe de enfermagem para executar suas práticas nos cenários do cuidado.

Os temas inferidos do material analisado apontam para a realização de registros de enfermagem cifrados, abreviados e com conteúdos que privilegiam o saber biomédico, configurando-se como uma problemática a ser pensada nas novas investigações que podem entrelaçar as especificidades de cada nível de complexidade dos serviços de saúde.

Como forma de superar os problemas identificados nas terminologias utilizadas pela enfermagem, os enfermeiros vêm pensando em estratégias que passam pela qualidade dos registros para assegurar a segurança do cuidado. Sinteticamente, observou-se: educação continuada; orientação sobre os registros de enfermagem in loco; adesão a protocolos; auditorias; e desenvolvimento de softwares para padronização da linguagem.

Generalizar os achados ainda não foi possível. Nesse aspecto, considera-se uma limitação a revisão não ser baseada estritamente em resultados de pesquisas clínicas, embora as teses incluídas atestem um rigor teórico e metodológico nos achados postos.

Com base nisso, o que emerge como propostas são: novas investigações sobre o fenômeno "registros de enfermagem" nos cenários de cuidar e revisões sistemáticas com o objetivo de comprovar ou refutar estes achados.

Assim, espera-se que este ensaio reflexivo seja capaz de suscitar novas posturas sobre a prática de pesquisar e registrar em enfermagem, beneficiando a profissão, os pacientes cuidados e a equipe multiprofissional em saúde nos diversos cenários do cuidado.

\section{REFERÊNCIAS}

1. Nightingale F. Notas sobre enfermagem: o que é e o que não é. Tradução de Amália Correa de Carvalho. São Paulo: Cortez; 1989.

2. Diniz SOS, Silva PS, Figueiredo NMA, Tonini T. Qualidade dos registros de enfermagem: reflexões analíticas em suas formas e conteúdos. Rev Enferm UFPE. 2015;9(10):9616-23.

3. Machado DA, Figueiredo NMA. Registros de enfermagem: a mensagem sobre o cuidado contida na linguagem escrita. Rev Pesq Cuid Fundam. 2010;2(Suppl.):293-7.
4. Meneghetti FK. O que é um ensaio-teórico? RAC. 2011;15(2):320-32.

5. Rother ET. Systematic literature review $X$ narrative review. Acta Paul Enferm. 2007;20(2)

http://dx.doi.org/10.1590/S0103-21002007000200001

6. Bardin L. Análise de conteúdo. Lisboa: Edições; 1977.

7. Japiassú $H$, Marcondes D. Dicionário básico de filosofia. Rio de Janeiro: Zahar; 2006. 
8. Conselho Federal de Enfermagem. Resolução COFEN nº 514 , de 5 de maio de 2016. Guia de Recomendações para os registros de enfermagem no prontuário do paciente e outros documentos de enfermagem. Brasília: COFEN; 2016.

9. Leonardi RCA. Avaliação dos aspectos éticos e legais dos registros de enfermagem na parada cardiorrespiratória em hospital escola do Paraná. Dissertação (Mestrado) - Escola de Enfermagem de Ribeirão Preto, Universidade de São Paulo. Ribeirão Preto: 2005.

10. Carvalho CJA, Nogueira MS. Análise dos registros de enfermagem em uma unidade cirúrgica de um hospital escola público. Rev Gaúcha Enferm. 2006;27(4):524-31.

11. Fontes $\mathrm{CMB}$. Perfis de diagnósticos de enfermagem antes e após a implementação da classificação da NANDA-I. Tese (Doutorado) Universidade de São Paulo. São Paulo: 2006.

12. Oguisso T. Dimensões ético-legais das anotações de enfermagem no prontuário do paciente. Rev Paul Enferm. 2003;22(3):245-54.

13. Diniz SOS. Modus operandi de registrar: diagnóstico situacional e propostas de intervenção. Dissertação (Mestrado) - Universidade Federal do Estado do Rio de Janeiro. Rio de Janeiro: 2013.

14. Machado DA. Registros de enfermagem: a mensagem sobre o cuidado contida na linguagem escrita. Dissertação (Mestrado) - Universidade Federal do Estado do Rio de Janeiro. Rio de Janeiro: 2010.

15. Reda E. Instrumento de registro utilizado na avaliação em sala de recuperação pós-anestésica: importância na continuidade da assistência ao paciente cirúrgico. Dissertação (Mestrado). Universidade de São Paulo. São Paulo: 2006.

16. Setz VG, D'Innocenzo M. Avaliação da qualidade dos registros de enfermagem no prontuário por meio da auditoria. Acta Paul Enferm. 2009;22(3):313-7.

17. Matsuda LM, Silva DMP, Évora YDM, Coimbra JAH. Anotações/ registros de enfermagem: instrumento de comunicação para a qualidade do cuidado. Rev Eletr Enf. 2006;8(3):415-21.

18. Gomes DC, Cubas MR, Pleis LE, Shmeil MAH, Peluci APVD. Termos utilizados por enfermeiros em registros de evolução do paciente. Rev Gaúcha Enferm. 2016;37(1):1-8. http://dx.doi.org/10.1590/1983-1447.2016.01.53927

19. Silva JA, Grossi ACM, Haddad MCL, Marcon SS. Avaliação da qualidade das anotações de enfermagem em unidade semiintensiva. Esc Anna Nery. 2012;16(3):576-81. http://dx.doi.org/10.1590/S1414-81452012000300021

20. Nomura ATG, Silva MB, Almeida MA. Qualidade dos registros de enfermagem antes e depois da Acreditação Hospitalar em um hospital universitário. Rev Latino-Am Enfermagem. 2016;24:e2813. http://dx.doi.org/10.1590/1518-8345.0686.2813

21. Fernández-Sola C, Granero-Molina J, Mollinedo-Mallea J, Gonzales MHP, Aguilera-Manrique G, Ponce ML. Desarrollo y validación de un instrumento para la evaluación inicial de enfermería. Rev Esc Enferm USP. 2012;46(6):1415-22. http://dx.doi.org/10.1590/S0080-62342012000600019 